\title{
Gorenstein Fano threefolds with base points in the anticanonical system
}

\author{
Priska Jahnke and Ivo Radloff
}

\begin{abstract}
We classify all Gorenstein Fano threefolds with at worst canonical singularities for which the anticanonical system $|-K|$ has a nonempty base locus.
\end{abstract}

\section{Introduction}

In the classification of Fano varieties, those which are not 'Gino Fano', i.e. for which $-K_{X}$ is ample but not very ample, are usually annoying. In the beginning of his classification of Fano threefolds Iskovskikh listed those for which $\left|-K_{X}\right|$ is not free (see [Isk78]). The purpose of this article is to see how his result extends to the canonical Gorenstein case.

If $X$ is a Gorenstein Fano threefold with at worst canonical singularities and $\mathrm{Bs}\left|-K_{X}\right| \neq \emptyset$, then the rational map defined by $\left|-K_{X}\right|$ goes to a surface $W$, which is a rational ruled surface $\Sigma_{e}$ with $e \geqslant 0$ or $\widehat{C}_{d}$, the cone over a rational normal curve of degree $d$. The following theorem lists the possible pairs $(X, W)$.

Theorem 1.1. Let $X$ be a Gorenstein Fano threefold with at worst canonical singularities and Bs $\left|-K_{X}\right| \neq \emptyset$. Then we are in one of the following cases.

(i) $\operatorname{dim} \operatorname{Bs}\left|-K_{X}\right|=0$. In this case $X$ is a complete intersection in $\mathbb{P}\left(1^{4}, 2,3\right)$ of a quadric $Q$, defined in the first four linear variables, and a sextic $F_{6} ;\left(-K_{X}\right)^{3}=2$ and $W$ is the quadric $Q$ in $\mathbb{P}_{3}$.

(ii) $\operatorname{dim} \operatorname{Bs}\left|-K_{X}\right|=1$. Then Bs $\left|-K_{X}\right| \simeq \mathbb{P}_{1}$ and:

(a) $X$ is the blowup of a sextic in $\mathbb{P}\left(1^{3}, 2,3\right)$ along a complete intersection curve of arithmetic genus $1,\left(-K_{X}\right)^{3}=4$ and $W \simeq \Sigma_{1}$; or

(b) $X \simeq S_{1} \times \mathbb{P}_{1}$, where $S_{1}$ is a del Pezzo surface of degree 1 with at worst Du Val singularities, $\left(-K_{X}\right)^{3}=6$ and $W \simeq \mathbb{P}_{1} \times \mathbb{P}_{1}$; or

(c) $X=X_{2 m-2}$ is an anticanonical model of the blowup of the variety $U_{m}$ (see below) along a smooth, rational complete intersection curve $\Gamma_{0} \subset U_{m, \text { reg }}$ for $3 \leqslant m \leqslant 12,\left(-K_{X}\right)^{3}=2 m-2$ and $W \simeq \widehat{C}_{m}$.

Here $U_{m}$ denotes a double cover of $\mathbb{P}\left(\mathcal{O}_{\mathbb{P}_{1}}(m) \oplus \mathcal{O}_{\mathbb{P}_{1}}(m-4) \oplus \mathcal{O}_{\mathbb{P}_{1}}\right)$ with at worst canonical singularities, such that $-K_{U_{m}}$ is the pullback of the tautological line bundle $\mathcal{O}(1)$. For $m \geqslant 4$, this is a hyperelliptic Gorenstein almost Fano threefold of degree $4 m-8$. The curve $\Gamma_{0}$ lies over the complete intersection of some general element in $|\mathcal{O}(1)|$ and the 'minimal surface' $B \in|\mathcal{O}(1)-m F|$, where $|F|$ denotes the pencil (note that $\Gamma_{0}$ is always contained in the ramification locus). If $m=3$, then $\Gamma_{0}$ is the only curve, on which $-K_{U_{3}}$ is not nef. For details of the construction, see $\S 5$.

The cases (a) and (b) are as in Iskovskikh's list. In a different context, case (i) appears in [Mel99] and [IT01], and apparently also in [Mor88].

Received 17 June 2004, accepted in final form 25 February 2005.

2000 Mathematics Subject Classification 14J45, 14J30.

Keywords: Fano varieties, threefolds.

This journal is (c) Foundation Compositio Mathematica 2006. 


\section{Gorenstein FAnO THREEFOLDS With BASE POINTS}

\section{Preliminaries}

We recall the following fundamental results.

Theorem 2.1 [Sho80, Rei83]. Let $X$ be a Gorenstein Fano threefold with at worst canonical singularities. Then $\left|-K_{X}\right|$ contains an irreducible surface $S$ with at worst Du Val singularities, called general elephant.

The birational contraction $h: Y \rightarrow X$ in the following theorem is called a partial crepant resolution or terminal modification of $X$.

Theorem 2.2 [Rei79, Kaw88]. Let $X$ be a threefold with only canonical singularities. Then there exists a $\mathbb{Q}$-factorial threefold $Y$ with only terminal singularities and a birational contraction $h: Y \rightarrow$ $X$ such that $K_{Y}=h^{*} K_{X}$.

If $X$ is Gorenstein, then $Y$ is, in fact, factorial (for example, [Kaw88, Lemma 5.1]).

A Gorenstein threefold $X$ for which $-K_{X}$ is big and nef is called almost Fano. It is called hyperelliptic, if $\left|-K_{X}\right|$ is free, but the associated map $\varphi$ fails to be injective at the generic point. In that case $\varphi: X \longrightarrow W \subset \mathbb{P}_{N}$ is generically two-to-one and $W$ is a so-called variety of minimal degree, i.e. $\operatorname{deg} W=$ codim $W+1$. Varieties of minimal degree have been classified by del Pezzo [delP85] in dimension 2 and by Bertini in arbitrary dimension $n$ (see [Ber07]). The list (with some repetitions) is as follows:

(i) $\mathbb{P}_{n}$;

(ii) the $n$-dimensional quadric $Q_{n} \subset \mathbb{P}_{n+1}$;

(iii) (a cone over) the Veronese surface;

(iv) (a cone over) a rational scroll.

The cone over a (rational) scroll, denoted by $\overline{\mathbb{F}\left(d_{1}, \ldots, d_{n}\right)}$, is the image of

$$
\mathbb{F}\left(d_{1}, \ldots, d_{n}\right)=\mathbb{P}\left(\mathcal{O}_{\mathbb{P}_{1}}\left(d_{1}\right) \oplus \cdots \oplus \mathcal{O}_{\mathbb{P}_{1}}\left(d_{n}\right)\right), \quad d_{1} \geqslant \cdots \geqslant d_{n} \geqslant 0
$$

in $\mathbb{P}_{d_{1}+\cdots+d_{n}+n-1}$ under the map associated with the tautological line bundle which will be denoted by $\mathcal{O}(1)$. Note that for $d_{n} \geqslant 1, \overline{\mathbb{F}\left(d_{1}, \ldots, d_{n}\right)}$ and $\mathbb{F}\left(d_{1}, \ldots, d_{n}\right)$ are isomorphic. The pencil on $\mathbb{F}\left(d_{1}, \ldots, d_{n}\right)$ will be denoted by $|F|$.

Any effective divisor $D$ on $\mathbb{F}\left(d_{1}, \ldots, d_{n}\right)$ is in a system $D \in|\mathcal{O}(k)-l F|, k \geqslant 0$ and $l \in \mathbb{Z}$. Fiberwise, $D \cap F$ is a hypersurface of degree $k$ in $\mathbb{P}_{n-1}$. If $x_{1}, \ldots, x_{n}$ denote homogeneous coordinates of $\mathbb{P}_{n-1}$ corresponding to the summands of our vector bundle, then the monomial $x_{1}^{e_{1}} \cdots x_{n}^{e_{n}}$ with $e_{1}+\cdots+e_{n}=k$ has as coefficient a function taken from $H^{0}\left(\mathbb{P}_{1}, \mathcal{O}_{\mathbb{P}_{1}}\left(e_{1} d_{1}+\cdots+e_{n} d_{n}-l\right)\right)$. We will use this in the following form. Consider $\mathbb{F}(m, m-4) \simeq \Sigma_{4}$. Denote by $\xi_{4}$ the minimal section. Any divisor

$$
D \in|\mathcal{O}(k)-l F|, \quad k \geqslant 0 \text { and } l>k(m-4)
$$

contains $\xi_{4}$ as a component. Indeed, using the above notation, $\xi_{4}$ corresponds fiberwise to $x_{1}=0$. It therefore suffices to prove that the coefficient function of $x_{2}^{k}$ vanishes. This is a section of $\mathcal{O}_{\mathbb{P}_{1}}(k(m-4)-l)$, so the claim follows.

\section{The general elephant in the case $\mathrm{Bs}\left|-K_{X}\right| \neq \emptyset$}

Let $X$ be a canonical Gorenstein Fano threefold with Bs $\left|-K_{X}\right| \neq \emptyset$. Choose a general elephant $\bar{S} \in\left|-K_{X}\right|$. By the Kawamata-Viehweg vanishing theorem $H^{0}\left(X,-K_{X}\right) \longrightarrow H^{0}\left(\bar{S},-\left.K_{X}\right|_{\bar{S}}\right)$ is surjective, implying Bs $\left|-K_{X}\right|=\operatorname{Bs}\left|-K_{X}\right|_{\bar{S}} \mid \neq \emptyset$. Let $\nu: S \rightarrow \bar{S}$ be a minimal desingularization 


\section{P. JAHNKE AND I. RADLOFF}

of $\bar{S}$. By Saint-Donat's results on linear systems on smooth K3 surfaces [Sai74, Shi89],

$$
\nu^{*}\left|-K_{X}\right| \bar{S}|=| \Gamma+m f \mid,
$$

where $m \geqslant 2$ and:

(i) $|f|$ is an elliptic pencil; and

(ii) $\Gamma=\operatorname{Bs}|\Gamma+m f| \simeq \mathbb{P}_{1}$ is a section.

Let $\Gamma^{\prime} \subset S$ be an irreducible curve contracted by $\nu$. Then $(\Gamma+m f) \cdot \Gamma^{\prime}=0$, implying $\Gamma \cap \Gamma^{\prime}=\emptyset$ or $\Gamma=\Gamma^{\prime}$. In the first case $S$ and $\bar{S}$ are isomorphic near $\Gamma$ and Bs $\left|-K_{X}\right| \simeq \mathbb{P}_{1} \subset \bar{S}_{\text {reg. In the second }}$

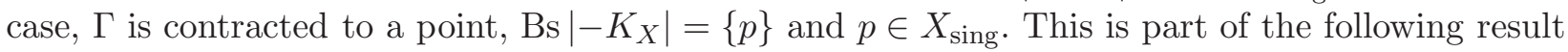
of Shin.

Theorem 3.1 [Shi89]. Let $X$ be a Gorenstein almost Fano threefold with at worst canonical singularities and assume $\mathrm{Bs}\left|-K_{X}\right| \neq \emptyset$. With $\bar{S} \in\left|-K_{X}\right|$ a general member we have:

(i) if $\operatorname{dim} \operatorname{Bs}\left|-K_{X}\right|=1$, then scheme-theoretically Bs $\left|-K_{X}\right| \simeq \mathbb{P}_{1}$ is contained in $X_{\text {reg }}$ and $\operatorname{Bs}\left|-K_{X}\right| \cap \operatorname{Sing}(\bar{S})=\emptyset$;

(ii) if $\operatorname{dim} \mathrm{Bs}\left|-K_{X}\right|=0$, then $\mathrm{Bs}\left|-K_{X}\right|$ consists of exactly one point and $\bar{S}$ has an ordinary double point at $\mathrm{Bs}\left|-K_{X}\right|$; in this case $\mathrm{Bs}\left|-K_{X}\right| \subset \operatorname{Sing}(X)$.

Note that in the case Bs $\left|-K_{X}\right|=\{p\}$ we have $(\Gamma+m f) \cdot \Gamma=0$ on $S$, implying $m=2$ and hence $\left(-K_{X}\right)^{3}=2$.

\section{The case $\operatorname{dim} \mathrm{Bs}\left|-K_{X}\right|=0$}

Let $X$ be the complete intersection of a quadric $Q$ in the linear variables and a sextic $F_{6}$ in $\mathbb{P}\left(1^{4}, 2,3\right)$. If we choose $F_{6}$ general enough, then (see [Mel99])

$$
X \cap\left\{x_{0}=x_{1}=x_{2}=x_{3}=0\right\}=[0: 0: 0: 0:-1: 1]=p
$$

and $X$ does not meet the singular locus of $\mathbb{P}\left(1^{4}, 2,3\right)$. Then $Q$ and $F_{6}$ are Cartier near $X$ and by adjunction, $-\left.K_{X} \simeq \mathcal{O}_{\mathbb{P}}(1)\right|_{X}$ and therefore Bs $\left|-K_{X}\right|=\{p\}$. The rational map defined by $\left|-K_{X}\right|$ sends $X$ to the quadric in $\mathbb{P}_{3}$ defined by $Q$.

Proposition 4.1. If $\operatorname{dim} \mathrm{Bs}\left|-K_{X}\right|=0$, then $X$ is as above a complete intersection in $\mathbb{P}\left(1^{4}, 2,3\right)$ of a quadric $Q$, defined in the first four linear variables, and a sextic $F_{6}$.

Proof. (See [Mor82, Mel99, IT01].) We know $\left(-K_{X}\right)^{3}=2$ (see $\left.\S 3\right)$. By the Riemann-Roch theorem we get $h^{0}\left(-K_{X}\right)=4$. Let $x_{0}, \ldots, x_{3} \in H^{0}\left(-K_{X}\right)$ be generating sections. We have $h^{0}\left(-2 K_{X}\right)=$ $10=\operatorname{dim} S^{2} H^{0}\left(-K_{X}\right)$. However, $\left|-2 K_{X}\right|$ is base point free, so there exists some

$$
y \in H^{0}\left(-2 K_{X}\right), \quad y \notin S^{2} H^{0}\left(-K_{X}\right) .
$$

Then we must have a nontrivial relation $Q$ in $S^{2} H^{0}\left(-K_{X}\right)$. The $x_{i}$ and $y$ then define a 20dimensional subspace of $H^{0}\left(-3 K_{X}\right)$. By the theorem of Riemann-Roch $h^{0}\left(-3 K_{X}\right)=21$. Denote the missing function by $z \in H^{0}\left(-3 K_{X}\right)$. Continuing in this way, we see that there must be a nontrivial relation $F_{6}$ in $H^{0}\left(-6 K_{X}\right)$. In the end $X$ is the complete intersection of $Q$ and $F_{6}$ in $\mathbb{P}\left(1^{4}, 2,3\right)$.

Remark 4.2. As $Q$ is singular at $p$, any $S \in\left|-K_{X}\right|$ is singular at $p$. If we choose $Q$ and $F_{6}$ general, $p$ will be a terminal point of $X$. If we take for $Q$ the quadric cone, $X$ will have canonical singularities along a curve. 


\section{Gorenstein FAno threefolds With Base points}

\section{The examples for the case $\operatorname{dim} \mathrm{Bs}\left|-K_{X}\right|=1$}

Let $U$ be a canonical Gorenstein threefold. Assume that $\left|-K_{U}\right|$ contains a smooth K3 surface $S$ such that $-\left.K_{U}\right|_{S}=2 \Gamma_{0}+m f$ for some $m \geqslant 3$. Here $\mathbb{P}_{1} \simeq \Gamma_{0} \subset U_{\text {reg }}$ and $|f|$ is an elliptic pencil as in $\S 3$. Note that $U$ is a hyperelliptic almost Fano threefold for $m \geqslant 4$.

Let $Y=B l_{\Gamma_{0}}(U)$ be the blowup of $U$ in $\Gamma_{0}$. The strict transform of $S$ is a smooth K3 surface in $\left|-K_{Y}\right|$ which we also denote by $S$. We have $-\left.K_{Y}\right|_{S}=\Gamma_{0}+m f$, implying Bs $\left|-K_{Y}\right|=\Gamma_{0} \simeq \mathbb{P}_{1}$. An anticanonical model $X$ of $Y$ is a canonical Gorenstein Fano threefold for which Bs $\left|-K_{X}\right| \simeq \mathbb{P}_{1}$.

Examples for $U$ as above are constructed as follows. For $m \geqslant 4, U$ is almost Fano and the anticanonical map associated with $-K_{U}$ sends $U$ to a variety of minimal degree $U \longrightarrow W \subset \mathbb{P}_{2 m-2}$. Here $S$ is sent to $\Sigma_{4}$, the fourth Hirzebruch surface. The idea is therefore to construct $U$ as a ramified twosheeted covering of some variety of minimal degree, for which a general hyperplane section is isomorphic to $\Sigma_{4}$.

We now come to the examples in Theorem 1.1(ii) in reverse order.

Example 1 (Theorem 1.1(ii)(c)). The projective bundle

$$
W=\mathbb{F}(m, m-4,0), \quad m \geqslant 3
$$

is a resolution of a cone over $\Sigma_{4}$. The projection of the underlying bundle onto the first two summands gives a split exact sequence and a smooth surface in $\left|\mathcal{O}_{W}(1)\right|$ isomorphic to $\Sigma_{4}$. For simplicity, we denote it by $\Sigma_{4} \in\left|\mathcal{O}_{W}(1)\right|$. There exists a unique section $B \in\left|\mathcal{O}_{W}(1)-m F\right|$ meeting $\Sigma_{4}$ in its minimal section $\xi_{4}$. Below we prove that for $m \leqslant 12$ we may choose $D \in\left|\mathcal{O}_{W}(4)-(4 m-12) F\right|$, such that the square root of $D$ yields a threefold $U_{m}$ with at worst canonical singularities. We have

$$
\mu: U_{m} \stackrel{2: 1}{\longrightarrow} \mathbb{F}(m, m-4,0) \quad \text { and } \quad-K_{U_{m}}=\mu^{*} \mathcal{O}_{W}(1)
$$

The section $\xi_{4}=B \cap \Sigma_{4} \subset D_{\text {reg. }}$. Its reduced inverse image in $U_{m}$ will be denoted by $\Gamma_{0}$. As in Theorem 1.1(ii)(c), we denote by $X_{2 m-2}$ an anticanonical model of $B l_{\Gamma_{0}}\left(U_{m}\right)$ for $3 \leqslant m \leqslant 12$. We claim that $X_{2 m-2}$ are canonical Gorenstein Fano threefolds with base locus Bs $\left|-K_{X_{2 m-2}}\right| \simeq \mathbb{P}_{1}$.

In order to prove this it suffices to show that for $D$ general enough each $U_{m}$ is a canonical Gorenstein threefold as in the beginning of this section. As $\Sigma_{4}$ comes from a splitting sequence, $D \cap \Sigma_{4}$ is a general member of $\left|4 \xi_{4}+12 \mathfrak{f}\right|$, with $\mathfrak{f} \simeq \mathbb{P}_{1}$ a fiber of $\Sigma_{4}$. A general member of $\left|4 \xi_{4}+12 \mathfrak{f}\right|$ splits as $\xi_{4}+C$ with $C \in\left|3 \xi_{4}+12 \mathfrak{f}\right|$ smooth and disjoint from $\xi_{4}$ (cf. $\S 2$ ). The double covering of $\Sigma_{4}$ yields a smooth K3 surface $S \in\left|-K_{U_{m}}\right|=\left|\mu^{*} \mathcal{O}_{W}(1)\right|$ with $\mu_{S}: S \longrightarrow \Sigma_{4}$ ramified along $\xi_{4}$ and $C$. The pullback of $\mathfrak{f}$ gives an elliptic pencil $|f|$ on $S$ with the section $\Gamma_{0}$ lying over $\xi_{4}$ and $-\left.K_{U_{m}}\right|_{S}=\mu_{S}^{*} \mathcal{O}(1)=2 \Gamma_{0}+m f$. It remains to show that $U_{m}$ has at worst canonical singularities for $3 \leqslant m \leqslant 12$ and $\Gamma_{0} \subset U_{m, \text { reg. }}$.

For $m=3$ we can choose $D$ and hence $U_{m}$ smooth and there is nothing to prove. For $m \geqslant 4$, we always have $D=B+R$ with $R \in\left|\mathcal{O}_{W}(3)-(3 m-12) F\right|$. Fiberwise $D \cap F$ consists of a line together with some cubic.

For $4 \leqslant m \leqslant 12$ we can take $R$ to be irreducible, i.e. $D \cap F$ consists of a line and an irreducible cubic. For $m=4$, the cubic is smooth, meeting the line transversally in three points. For $m \geqslant 5$, the line and the cubic intersect in one point, i.e. in a flex if the cubic is smooth. This gives an A-D-E singularity in the fiber, implying that $U_{m}$ indeed has at worst canonical singularities for $3 \leqslant m \leqslant 12$. As $R . \xi_{4}=0$ we can choose $R$ disjoint from $\xi_{4}$. Hence, $\Gamma_{0} \subset U_{m, \text { reg }}$.

For $m \geqslant 13$, on the other hand, $R=R_{1}+R_{2}+R_{3}$ with $R_{i} \in\left|\mathcal{O}_{W}(1)-(m-4) F\right|$, so $D \cap F$ consists of four lines through a point. This means that over $F$ we will not have Du Val singularities, implying that $U_{m}$ is not canonical for $m \geqslant 13$.

Remark 5.1. The construction also works for $m=2$. Here Bs $\left|-K_{X_{2}}\right|=\{p\}$ and we get a special case of the threefold $X$ in $\S 4$ with $Q$ the quadric cone (see Remark 4.2). 


\section{P. JAHNKE AND I. RADLOFF}

Example 2 (Theorem 1.1(ii)(b)). The product of $S_{1}$, a del Pezzo surface with canonical singularities of degree 1 , and $\mathbb{P}_{1}$ is a classical example [Isk80]. Choose eight points on $\mathbb{P}_{2}$ general enough, such that the blowup $\hat{\mathbb{P}}_{2}$ of $\mathbb{P}_{2}$ in these points still has a nef anticanonical system, and denote by $S_{1}$ an anticanonical model of $\hat{\mathbb{P}}_{2}$. Then $\left|-K_{S_{1}}\right|$ is one dimensional by the Riemann-Roch theorem, its members corresponding to elliptic curves passing through the eight points. These curves will meet in a ninth point, implying Bs $\left|-K_{S_{1}}\right|=\{p\}$. Then the product $X=S_{1} \times \mathbb{P}_{1}$ is a canonical Gorenstein Fano threefold with $\mathrm{Bs}\left|-K_{X}\right| \simeq \mathbb{P}_{1}$.

Example 3 (Theorem 1.1(ii)(a)). The blowup $X$ in the intersection of two members of $\left|-\frac{1}{2} K_{U}\right|$ of the double cover $U$ of the Veronese cone $W$, ramified along a cubic, is a classical example [Isk80]. We give some details to show the connection to the above description.

The blowup of the Veronese cone in its vertex $O$ yields $\mathbb{P}\left(\mathcal{O}_{\mathbb{P}_{2}} \oplus \mathcal{O}_{\mathbb{P}_{2}}(2)\right) \longrightarrow W$. The strict transform of a special hyperplane section through $O$ gives a $\mathbb{P}_{1}$-bundle over a conic. It either decomposes into two copies of $\Sigma_{2}$ or gives one irreducible surface $\Sigma_{4}$.

The image of $\Sigma_{4}$ in $W$ gives $\widehat{C}_{4}$, the cone over the rational normal curve of degree 4 . In $U$, lying over $\widehat{C}_{4}$ we find a singular K3 surface $\bar{S} \in\left|-K_{U}\right|$ with a double point over $O$. In the reducible case, the two copies of $\Sigma_{2}$ induce $H_{i} \in\left|-\frac{1}{2} K_{U}\right|$ for $i=1,2$, and their intersection with $\bar{S}$ is the singular point.

In the blowup $X$ of $U$ along $H_{1} \cap H_{2}$ the singularity of $\bar{S}$ is resolved, i.e. we get a smooth K3 surface $S \in\left|-K_{X}\right|$. The same formulas as above show $-\left.K_{X}\right|_{S}=\Gamma+2 f$ with $\Gamma$ the -2 -curve over the singularity and $|f|$ the induced elliptic pencil. If we choose $H_{1}, H_{2}$ general enough, then $X$ will be a canonical Gorenstein Fano threefold with $\mathrm{Bs}\left|-K_{X}\right| \simeq \Gamma \simeq \mathbb{P}_{1}$.

\section{The general setting in the case $\operatorname{dim} B s\left|-K_{X}\right|=1$}

We now look at the general setting in the case $\operatorname{dim} \operatorname{Bs}\left|-K_{X}\right|=1$ (cf. [Isk80, IP99]). By Shin's Theorem, $\Gamma=\operatorname{Bs}\left|-K_{X}\right| \simeq \mathbb{P}_{1} \subset X_{\text {reg. }}$. We can write

$$
N_{\Gamma / X}=\mathcal{O}_{\mathbb{P}_{1}}(a) \oplus \mathcal{O}_{\mathbb{P}_{1}}(b), \quad a \geqslant b,
$$

for some $a, b \in \mathbb{Z}$. A general elephant $\bar{S} \in\left|-K_{X}\right|$ may have double points, but $\Gamma \subset \bar{S}_{\text {reg. }}$. If $\nu: S \rightarrow \bar{S}$ denotes a resolution of the singular locus, then $\nu^{*}\left(-K_{X}\right)=\Gamma+m f, m \geqslant 3$, with $|f|$ an elliptic pencil and $\Gamma$ a section $(\S 3)$. The numbers are related as follows:

$$
-K_{X} \cdot \Gamma=m-2=a+b+2 .
$$

Let $\sigma: X_{\Gamma} \rightarrow X$ be the blowup of $X$ along $\Gamma$ with exceptional divisor $E_{\Gamma}=\mathbb{P}\left(N_{\Gamma / X}^{*}\right)=\Sigma_{a-b}$. Then $\left|-K_{X_{\Gamma}}\right|=\left|\sigma^{*}\left(-K_{X}\right)-E_{\Gamma}\right|$ is free, defining a map onto some surface $W$ (see [Rei83]).

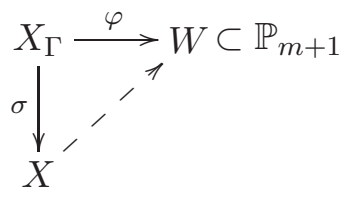

The surface $W$ is of minimal degree, i.e. $m=\operatorname{deg}(W)=\operatorname{codim}(W)+1$. Again by del Pezzo's theorem, in our situation $W$ is one of the following:

(i) $\widehat{C}_{m}$, the cone over a rational normal curve of degree $m=a+b+4 \geqslant 2$; or

(ii) $\Sigma_{a-b}, a \geqslant b$.

The map $E_{\Gamma} \rightarrow W$ is either an isomorphism or the contraction of the minimal section. The map $X_{\Gamma} \rightarrow W$ is (generically) an elliptic fibration, and as $-K_{X}$ is ample, any fiber over a point in $W_{\text {reg }}$ is an irreducible, generically reduced curve of arithmetic genus one. We distinguish two cases. 


\section{The case $W$ a smooth ruled surface}

Here we denote by $F_{\Gamma}$ the pullback to $X_{\Gamma}$ of a fiber of $W$, and by $Z_{\Gamma, X}$ the pullback of the minimal section (or the second ruling in the case $W=\mathbb{P}_{1} \times \mathbb{P}_{1}$ ). Note that $\left|F_{\Gamma}\right|$ descends to a pencil $|F|$ on $X$. Adjunction on $E_{\Gamma}$ shows $-K_{X_{\Gamma}}=Z_{\Gamma, X}+(a+2) F_{\Gamma}$. As $\Gamma \subset X_{\text {reg }}$ and $Z_{\Gamma, X}$ meets $E_{\Gamma}$ transversally near the minimal section $\xi_{a-b}$ of $E_{\Gamma}, Z_{\Gamma, X}$ is smooth near $Z_{\Gamma, X} \cap E_{\Gamma}$, and $\sigma\left(Z_{\Gamma, X}\right) \simeq Z_{\Gamma, X}$ is smooth near $\Gamma$.

\section{The case $W$ a cone}

Here we denote by $F_{\Gamma}$ the strict transform in $X_{\Gamma}$ of a line in $W$ through the vertex $O$. Note that this is just a Weil divisor. Let

$$
h^{\prime}: X_{\Gamma}^{\prime} \longrightarrow X_{\Gamma}
$$

be a $\mathbb{Q}$-factorialization of $X_{\Gamma}$ with respect to $F_{\Gamma}$ (see [Kaw88]). The map $h^{\prime}$ is small, $X_{\Gamma}^{\prime}$ is again Gorenstein with at worst canonical singularities, and the strict transform $F_{\Gamma}^{\prime}$ of $F_{\Gamma}$ is $\mathbb{Q}$-Cartier. We can choose $X_{\Gamma}^{\prime}$ such that $F_{\Gamma}^{\prime}$ is $h^{\prime}$-ample [Kaw88]. As $\Gamma \subset X_{\text {reg }}$, both $X_{\Gamma}^{\prime}$ and $X_{\Gamma}$ are isomorphic near $E_{\Gamma}$. We denote the pullback of $E_{\Gamma}$ to $X_{\Gamma}^{\prime}$ by $E_{\Gamma}^{\prime}$. We claim (cf. [Che99]) the following.

Lemma 6.1. On $X_{\Gamma}^{\prime}$, two general members of $\left|F_{\Gamma}^{\prime}\right|$ do not intersect.

Proof. Assume $F_{\Gamma, 1}^{\prime} \cap F_{\Gamma, 2}^{\prime} \neq \emptyset$. The intersection is clearly in the fiber over the vertex $O$ of $W$. Choose an irreducible curve $C \subset F_{\Gamma, 1}^{\prime} \cap F_{\Gamma, 2}^{\prime}$. On the one hand, the restriction of some multiple of $F_{\Gamma, 2}^{\prime}$, which is Cartier, gives an effective Cartier divisor on $F_{\Gamma, 1}^{\prime}$ supported in the fiber over $O$, implying $F_{\Gamma, 2}^{\prime} \cdot C \leqslant 0$. On the other hand, as $F_{\Gamma, 1}^{\prime}$ and $F_{\Gamma, 2}^{\prime}$ do not meet on $E_{\Gamma}^{\prime}$, we have $C \cap E_{\Gamma}^{\prime}=\emptyset$. As $-K_{X_{\Gamma}^{\prime}} \cdot C=0$ and $E_{\Gamma}^{\prime} \cdot C=0$ imply $h^{\prime *} \sigma^{*}\left(-K_{X}\right) \cdot C=0$, the curve $C$ must be $h^{\prime}$-exceptional. Then, by our choice of $X_{\Gamma}^{\prime}$, we have $F_{\Gamma, 2}^{\prime} \cdot C>0$. Hence, $F_{\Gamma, 1}^{\prime} \cap F_{\Gamma, 2}^{\prime}=\emptyset$.

Denote by $Y_{\Gamma}$ a terminal modification of $X_{\Gamma}^{\prime}$. The pullback of $F_{\Gamma}^{\prime}$ to $Y_{\Gamma}$ defines a pencil on $Y_{\Gamma}$, showing that the map to $W$ factors over the blowup $\Sigma_{a-b}$ of $W$ in $O$. Near $E_{\Gamma}^{\prime}, Y_{\Gamma}$ and $X_{\Gamma}^{\prime}$ are isomorphic, and we can blow the divisor down to obtain $Y$, a terminal modification $h: Y \rightarrow X$ of $X$. We call the map $Y_{\Gamma} \rightarrow Y$ again $\sigma$ and end up with the following diagram.

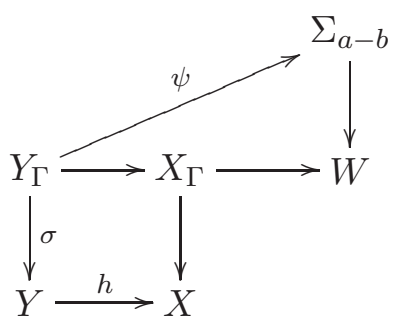

Below, we study $Y$ instead of $X$ and think of $X$ as an anticanonical model. Note that we have chosen $Y$ as a terminal modification of a particular $\mathbb{Q}$-factorialization of $X$.

For simplicity, denote divisors on $Y_{\Gamma}$ and $X_{\Gamma}$ by the same letters: the exceptional divisor of $Y_{\Gamma} \rightarrow Y$ is again $E_{\Gamma}$, the curve Bs $\left|-K_{Y}\right|=\Gamma$. The pullback of a general fiber of $\Sigma_{a-b}$ to $Y_{\Gamma}$ is $F_{\Gamma}$. By $Z_{\Gamma}+B_{\Gamma}$ we denote the pullback of the minimal section of $\Sigma_{a-b}$ to $Y_{\Gamma}$, where $Z_{\Gamma}$ denotes here the unique irreducible component that meets $E_{\Gamma}$ in its minimal section, and $B_{\Gamma}$ consists of the remaining components, disjoint from $E_{\Gamma}$. As above we get

$$
-K_{Y_{\Gamma}}=Z_{\Gamma}+B_{\Gamma}+(a+2) F_{\Gamma} .
$$

The pencil $\left|F_{\Gamma}\right|$ again descends to the pencil $|F|$ on $Y$. The surface $Z_{\Gamma}$ is smooth near $E_{\Gamma} \cap Z_{\Gamma}$; we will denote the isomorphic images of $Z_{\Gamma}$ and $B_{\Gamma}$ in $Y$ by $Z$ and $B$. 


\section{P. JAHNKE AND I. RADLOFF}

Remark 6.2. The general member of the pencil $\left|F_{\Gamma}\right|$ is a smooth surface with a relatively minimal elliptic pencil. The intersection $F_{\Gamma} \cap\left(Z_{\Gamma}+B_{\Gamma}\right)$ is hence either smooth or one of Kodaira's exceptional fibers.

\section{The case $W$ a cone}

Proposition 7.1. If $W$ is a cone, then $3 \leqslant m \leqslant 12$ and $X=X_{2 m-2}$ is one of the threefolds constructed in Example 1. Here $W=\widehat{C}_{m}$.

Proof. We use the notation from the last section. As $-K_{X_{\Gamma}}$ is not ample on $E_{\Gamma}, b=-2$ and $a \geqslant 1$ in (6.0.1). We can hence use $a+b=m-4$ to eliminate $a$ and $b$ and write everything in terms of $m$ :

$$
N_{\Gamma / X}=\mathcal{O}_{\mathbb{P}_{1}}(m-2) \oplus \mathcal{O}_{\mathbb{P}_{1}}(-2), \quad m \geqslant 3,
$$

and $W=\widehat{C}_{m}$. In diagram (6.1.1), the map from $Y_{\Gamma}$ to $\widehat{C}_{m}$ now factors over $\Sigma_{m}$.

We first assume that $Z$ is $h$-nef and show that in this case $Y$ is obtained by blowing up some Gorenstein threefold $V$ along some smooth curve $\Gamma_{0} \simeq \mathbb{P}_{1} \subset V_{\text {reg, }}$, such that $Z$ is the exceptional divisor. We compute $Z \cdot \Gamma=-2$ and $-K_{Y} \cdot \Gamma=m-2>0$. Hence $[\Gamma]$ is contained in the $K_{Y}$-negative part of $\overline{N E}(Y)$. This part is polyhedral, spanned by $K_{Y}$-negative extremal rays. The divisor $Z$ is negative on $[\Gamma]$ and nonnegative on any $K_{Y}$-trivial curve by assumption. We conclude that $Z$ must be negative on at least one extremal ray. Let

$$
\phi: Y \longrightarrow V
$$

be the contraction of this ray. By [Ben85], the contraction is divisorial, contracting $Z$ either to a curve or to a point. We claim the following.

Lemma 7.2. The map $\phi: Y \rightarrow V$ in (7.1.1) is the blowup of a smooth rational curve $\Gamma_{0} \subset V_{\text {reg }}$ with normal bundle $N_{\Gamma_{0} / V}=\mathcal{O}_{\mathbb{P}_{1}}(-2) \oplus \mathcal{O}_{\mathbb{P}_{1}}(m-4)$. The contraction is in direction of $|F|$. There exists a smooth $K 3$ surface $S \in\left|-K_{V}\right|$, such that $-\left.K_{V}\right|_{S}=2 \Gamma_{0}+m f$ with $|f|$ an elliptic pencil induced by $|F|$, and $\Gamma_{0} \simeq \mathbb{P}_{1}$ a smooth section.

Remark 7.3. The threefold $V$ is a hyperelliptic Gorenstein almost Fano threefold of degree $\left(-K_{V}\right)^{3}=$ $4 m-8$ for $m \geqslant 4$. For $m=3$, the anticanonical system is nef on any curve $\neq \Gamma_{0}$, while $-K_{V} \cdot \Gamma_{0}=$ $m-4=-1$. For the case $m=3$ (as well as $m=2$ ), see also [DPS93].

Proof of Lemma 7.2 and Remark 7.3. As $Z_{\Gamma}$ meets $E_{\Gamma}$ transversally in the minimal section, we have $\Gamma \subset Z_{\text {reg. }}$ We compute

$$
\operatorname{deg} N_{\Gamma / Z}=Z_{\Gamma} \cdot Y_{\Gamma} E_{\Gamma}^{2}=m-2>0 .
$$

Let us first show $Z \not \mathbb{P}_{1} \times \mathbb{P}_{1}$. If $Z \simeq \mathbb{P}_{1} \times \mathbb{P}_{1}$, then $B \neq 0$, implying that $B$ meets $Z$ in some curve. By (7.3.1) $\Gamma$ is ample on $Z$. Then $\Gamma \cap B \neq \emptyset$, which is impossible as $B$ maps to $X_{\text {sing, while }}$ $\Gamma \subset X_{\text {reg. }}$

If $Z$ is mapped to a point, then by [Cut88], $Z \simeq \mathbb{P}_{2}, \mathbb{P}_{1} \times \mathbb{P}_{1}$ or the quadric cone. As $Z$ comes with a pencil and $Z \not \mathbb{P}_{1} \times \mathbb{P}_{1}$, all of these cases are impossible. By [Cut88], $Y=B l_{\Gamma_{0}}(V)$ the blowup of $V$ in some curve $\Gamma_{0} \subset V_{\text {reg }}$, which is locally a complete intersection. From $\operatorname{deg} N_{\Gamma / Z}=m-2>0$ we conclude that $\Gamma$ maps surjectively onto $\Gamma_{0}$, and from $\Gamma \subset Z_{\text {reg }}$ we infer that $\Gamma_{0}$ must be smooth. Then $Z=\mathbb{P}\left(N_{\Gamma_{0} / V}^{*}\right) \simeq \Sigma_{e}$ for some $e>0$, where $e>0$ follows from $Z \neq \mathbb{P}_{1} \times \mathbb{P}_{1}$. It is now clear that $\phi$ is in the direction of $|F|$, i.e. fiberwise $\phi$ contracts a -1-curve in $F$. Denote the induced pencil on $V$ by $\left|F_{V}\right|$. Note that $Z \simeq \Sigma_{e}$ implies $B \neq 0$.

(1) Any curve in $Z_{\Gamma} \cap B_{\Gamma}$ is contracted by $Y_{\Gamma} \rightarrow X_{\Gamma}$, and therefore $B$ intersects $Z$ set theoretically in the minimal section $\xi_{e}$ of $Z=\Sigma_{e}$. As $\Gamma$ does not meet $\xi_{e}$, we conclude $\Gamma=\xi_{e}+(m-2) \mathfrak{f}_{e}$, where $\mathfrak{f}_{e}$ 


\section{Gorenstein FAno threefolds With Base points}

is a fiber of $\Sigma_{e}$. From $\Gamma \cdot{ }_{Z} \Gamma=m-2\left(\right.$ see (7.3.1)) we infer $e=m-2$. Moreover, $-K_{Y} \cdot \xi_{e}=0$ implies $N_{\Gamma_{0} / V}=\mathcal{O}_{\mathbb{P}_{1}}(-2) \oplus \mathcal{O}_{\mathbb{P}_{1}}(m-4)$. By the adjunction formula, $-K_{V} \cdot \Gamma_{0}=m-4$, hence $\left(-K_{V}\right)^{3}=4 m-8$.

(2) Let $S \in\left|-K_{Y}\right|$ be general. As $S$ meets $Z$ transversally in $\Gamma$, its image in $V$ is a special member of $\left|-K_{V}\right|$. Identifying $S$ with its image in $V$ we find $-\left.K_{V}\right|_{S}=2 \Gamma_{0}+m f$, where $|f|$ is an elliptic pencil and $\Gamma_{0}$ is a section (see $\S 5$ ). If $C \subset V$ is an irreducible curve such that $-K_{V} \cdot C<0$, then $S \cdot C<0$ and $C \subset S$. Then $-K_{V} \cdot C=\left(2 \Gamma_{0}+m f\right) \cdot C<0$ so that $\Gamma_{0} \cdot C<0$ and hence $C=\Gamma_{0}, m=3$.

The argument before Lemma 7.2 showing the contractibility of $Z$ in $Y$ requires $Z$ being $h$-nef. In order to achieve this we might have to change the terminal modification by running the relative $\left(K_{Y}+\epsilon Z\right)$-program, $\epsilon \in \mathbb{Q}^{+}, \epsilon \ll 1$, with respect to $h: Y \rightarrow X$.

The contraction of any $\left(K_{Y}+\epsilon Z\right)$-negative extremal ray in $\overline{N E}(Y / X)$ is small; the curves contracted are $K_{Y}$-trivial and contained in $Z$. After finitely many flops, we end up with the following diagram [KM98, Theorem 6.14 and Corollary 6.19].

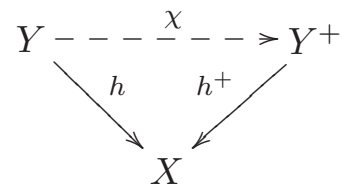

Here $Y^{+}$is again a terminal Gorenstein threefold with $-K_{Y^{+}}$big and nef, having $X$ as an anticanonical model. The map $\chi$ is rational and an isomorphism in codimension one. We superscribe any strict transform under $\chi$ with a ' ${ }^{\prime}$ ' sign. As $K_{Y^{+}}+\epsilon Z^{+}$is $h^{+}$-nef, $Z^{+}$is $h^{+}$-nef. As above we conclude that $Z^{+}$is contractible in $Y^{+}$.

Lemma 7.2 holds for $Y^{+}$instead of $Y$ as long as $\left|F^{+}\right|$is still spanned on $Y^{+}$. This need not be the case. Recall that we have chosen $Y$ as a terminal modification of some $\mathbb{Q}$-factorialization $X^{\prime}$ of $X$; in the above program we might flop some horizontal curves in $Z$, thereby producing a base locus.

Lemma 7.4. The system $\left|F^{+}\right|$is spanned unless $m=3$ and $\left(Z^{+}, \mathcal{O}_{Z^{+}}\left(Z^{+}\right)\right)=\left(\mathbb{P}_{2}, \mathcal{O}_{\mathbb{P}_{2}}(-2)\right)$.

Here $\left|F^{+}\right|$restricted to $Z^{+}$corresponds to lines through a given point.

Proof. Assume that $\left|F^{+}\right|$is not spanned. Let $\phi^{+}: Y^{+} \longrightarrow V^{+}$be the divisorial contraction as in (7.1.1), contracting $Z^{+}$. In order to decide what $Z^{+}$is, we again use the classification from [Cut88]. If $Z^{+}$maps to a curve and $\mathfrak{f}$ denotes the general fiber, then $Z^{+} \cdot \mathfrak{f}=-1$ and $-K_{Y^{+}} \cdot \mathfrak{f}=1$. On $Y^{+}$ we have

$$
-K_{Y^{+}}=Z^{+}+B^{+}+m F^{+} \text {. }
$$

As Bs $\left|F^{+}\right| \cap Z^{+} \neq \emptyset$ we must have $F^{+} \cdot \mathfrak{f}>0$. From $B^{+} \cdot \mathfrak{f} \geqslant 0$ we conclude that $0<m \mathfrak{f} \cdot F^{+} \leqslant 2$, which is impossible as $m>2$.

If $Z^{+}$goes to a point, then $\left(Z^{+}, \mathcal{O}_{Z^{+}}\left(Z^{+}\right)\right)$is either $\left(\mathbb{P}_{2}, \mathcal{O}(-1)\right)$ or $\left(\mathbb{P}_{2}, \mathcal{O}(-2)\right)$ or $\left(Q_{2} \subset\right.$ $\left.\mathbb{P}_{3}, \mathcal{O}(-1)\right)$. Near $\Gamma$ the two surfaces $Z$ and $Z^{+}$are isomorphic. With the original pencil on $Z$ we conclude that $Z^{+}$contains a smooth rational curve that meets another irreducible curve in a single point. From $Z^{+} \cdot \Gamma=-2$ we infer $\left(Z^{+}, \mathcal{O}_{Z^{+}}\left(Z^{+}\right)\right)=\left(\mathbb{P}_{2}, \mathcal{O}(-2)\right)$. Then $\left|F^{+}\right|$restricted to $Z^{+}$is a family of lines. Using $-K_{Y^{+}} \cdot \Gamma=m-2$ and the adjunction formula, we find $m=3$. The proof of the lemma is complete.

Lemma 7.2 also holds in the exceptional case $\left(Z^{+}, \mathcal{O}_{Z^{+}}\left(Z^{+}\right)\right)=\left(\mathbb{P}_{2}, \mathcal{O}_{\mathbb{P}_{2}}(-2)\right)$ for some terminal modification of $X^{\prime}$, we only cannot argue as above. Instead, we proceed as follows. 


\section{P. JAHNKE AND I. RADLOFF}

We first run the relative $\left(K_{Y}+\epsilon Z\right)$-program with respect to $Y \rightarrow X^{\prime}$, where $X^{\prime}$ is the above $\mathbb{Q}$-factorialization of $X$. In the end we may assume that $Z$ is at least nef on every $K_{Y}$-trivial curve contained in a fiber of the pencil $Z \rightarrow \mathbb{P}_{1}$. Omitting some details, we conclude that a single flop of a $K_{Y}$-trivial section of $Z$ transforms $Y$ into $Y^{+}$in (7.3.2) and $Z$ into $Z^{+}=\mathbb{P}_{2}$ as above. Then $Z \simeq \Sigma_{1}$ and $Z \cdot \mathfrak{f}=-1$ for the general fiber $\mathfrak{f} \simeq \mathbb{P}_{1}$. We conclude that $Z$ must be negative on at least one extremal ray in $\overline{N E}\left(Y / \mathbb{P}_{1}\right)$ and conclude Lemma 7.2 as above.

For the proof of Proposition 7.1 it remains to show that $V$ in Lemma 7.2 is a terminal modification of $U_{m}$ in $\S 5$. In order to prove this, we consider the system $\left|-K_{V}+\lambda F_{V}\right|, \lambda \geqslant 0$, and choose $\lambda$ such that $m+\lambda \geqslant 4$. Restricted to $S$ we get $2 \Gamma_{0}+(m+\lambda) f$, which is now big and nef. Then $-K_{V}+\lambda F_{V}$ is big and nef and by the Kawamata-Viehweg vanishing theorem $H^{1}\left(\mathcal{O}_{V}\left(\lambda F_{V}\right)\right)=H^{1}\left(\mathcal{O}_{V}\left(K_{V}+\right.\right.$ $\left.\left.\left(-K_{V}+\lambda F_{V}\right)\right)\right)=0$ implying surjectivity of

$$
H^{0}\left(V, \mathcal{O}_{V}\left(-K_{V}+\lambda F_{V}\right)\right) \longrightarrow H^{0}\left(S, \mathcal{O}_{S}\left(2 \Gamma_{0}+(m+\lambda) f\right)\right) .
$$

Then, as $\left|F_{V}\right|$ is free and $\left|2 \Gamma_{0}+(m+\lambda) f\right|$ is free, $\left|-K_{V}+\lambda F_{V}\right|$ is free. For $\lambda \geqslant 1$ and $m+\lambda \geqslant 5$, any irreducible curve having zero intersection with $-K_{V}+\lambda F_{V}$ must lie in a member of $\left|F_{V}\right|$. This follows immediately from $-K_{V}+\lambda F_{V}=\left(-K_{V}+(\lambda-1) F_{V}\right)+F_{V}=$ nef + nef. The system is free, for example, if we choose $\lambda=1$, for $m \geqslant 4$, and $\lambda=2$, for $m=3$.

Fix this choice from now on. The map associated with $\left|-K_{V}+\lambda F_{V}\right|$ is generically two-toone sending $V$ to a variety of minimal degree $\nu: V \longrightarrow W \subset \mathbb{P}_{2 m+3 \lambda-2}$. As $W$ comes with a pencil $\left|F_{W}\right|$, it must be a scroll. We may rescale the entries such that $-K_{V} \simeq \nu^{*} \mathcal{O}_{W}(1)$. Then $W \simeq \mathbb{F}\left(d_{1}, d_{2}, d_{3}\right), d_{1} \geqslant d_{2} \geqslant d_{3} \geqslant-1$, where $d_{3}=-1$ in the case $m=3$, while $d_{3} \geqslant 0$ for $m \geqslant 4$. Stein factorization of $V \rightarrow W$ leads to a canonical Gorenstein threefold $U$ and a double cover $\mu: U \longrightarrow W \simeq \mathbb{F}\left(d_{1}, d_{2}, d_{3}\right)$, such that $-K_{U}=\mu^{*} \mathcal{O}_{W}(1)$. Hence, $\mu$ is ramified along a reduced divisor $D \in\left|\mathcal{O}_{W}(4)-2\left(d_{1}+d_{2}+d_{3}-2\right) F_{W}\right|$. From $\left(\mathcal{O}_{W}(1)\right)^{3}=\frac{1}{2}\left(-K_{V}\right)^{3}=2 m-4$ we infer $d_{1}+d_{2}+d_{3}=2 m-4$. The only section of $H^{0}\left(V,-K_{V}-m F_{V}\right)$ is that corresponding to the image of $B$ in $V$ (cf. (6.1.2)). As $\mu$ is fiberwise ramified along a quartic, we also have $h^{0}\left(W, \mathcal{O}_{W}(1)-m F_{W}\right)=1$, implying $d_{1}=m, d_{2}<m$. In the special case $m=3$ we have $d_{3}=-1$ and $W \simeq \mathbb{F}(3,0,-1)$. It remains to consider the case $m \geqslant 4$.

Denote the image of $B$ in $W$ by $B_{W}$. If $d_{3}>0$, then $2 B_{W}$ is a component of $D$. However $D$ is reduced, hence we must have $d_{3}=0$. Then $d_{1}=m, d_{2}=m-4$, i.e. $V \longrightarrow U \longrightarrow W \simeq \mathbb{F}(m, m-4,0)$. We have seen in $\S 5$, that $U=U_{m}$ can never have canonical singularities for $m \geqslant 13$, hence $m \leqslant 12$.

Back on the surface $S \in\left|-K_{V}\right|$ in Lemma 7.2, we see that $S$ is generically a double cover of some member $H \in\left|\mathcal{O}_{W}(1)\right|$. The map $\nu$ sends $S$ to $\mathbb{F}(m, m-4)$ and $\Gamma_{0}$ lies over the minimal section, which is the restriction of the above divisor $B_{W}$. In particular, $\Gamma_{0}$ is not contracted by $V \rightarrow U_{m}$ and does not meet any curve contracted, i.e. $\Gamma_{0} \subset U_{m, \text { reg }}$ and $V$ is isomorphic to $U_{m}$ near $\Gamma_{0}$. This completes the proof of Proposition 7.1.

\section{The case $W$ a ruled surface}

This case is as in [Isk80]. Instead of $Y$ and $Y_{\Gamma}$ we focus on $X$ and $X_{\Gamma}$, and diagram (6.0.2). We use the notation introduced in $\S 6$.

Proposition 8.1. In the case $W \simeq \Sigma_{a-b}, a>b, X$ is the blowup of a sextic in $\mathbb{P}\left(1^{3}, 2,3\right)$ along an irreducible curve of arithmetic genus one (and $a=0, b=-1, m=3$ ).

Proof. As $-K_{X_{\Gamma}}$ is ample on $E_{\Gamma}$, we have $b \geqslant-1$ and $a \geqslant 0$. Hence,

$$
Z_{\Gamma, X} \cdot \xi_{a-b}=b-a<0 \text { and }-K_{X_{\Gamma}} \cdot \xi_{a-b}=b+2>0,
$$

where $\xi_{a-b}=E_{\Gamma} \cap Z_{\Gamma, X}$ is the minimal section of $E_{\Gamma}$. As $Z_{\Gamma, X}$ is trivial on any $K_{X_{\Gamma}}$-trivial curve, we conclude that $Z_{\Gamma, X}$ must be negative on at least one extremal ray in $\left\{K_{X_{\Gamma}}<0\right\}$. 


\section{Gorenstein FAno threefolds With Base points}

Denote by $\phi_{X}: X_{\Gamma} \longrightarrow V_{X}$ the contraction of this ray. It is a birational map with exceptional set $Z_{\Gamma, X}$ by [Ben85]. As $Z_{\Gamma, X}$ contains $K_{X_{\Gamma}}$-trivial curves, it is contracted to a curve.

If $Z_{\Gamma, X}$ is singular along a curve, then its normalization is a smooth ruled surface. The second map implies that it is $\mathbb{P}_{1} \times \mathbb{P}_{1}$. As $\xi_{a-b} \subset Z_{\Gamma, X \text {,reg }}$ does not meet the singular locus, we must have $\operatorname{deg} N_{\xi_{a-b} / Z_{\Gamma, X}}=a=0$, implying $b=-1$. If $Z_{\Gamma, X}$ is smooth in codimension one, then $h^{1}\left(Z_{\Gamma, X}, \mathcal{O}_{Z_{\Gamma, X}}\right) \leqslant 1$ by [Rei83] and Iskovskikh's original argument applies: using the ideal sequence of $Z_{\Gamma, X}$ and the identity $-K_{X_{\Gamma}}=Z_{\Gamma, X}+(a+2) F_{\Gamma}($ cf. $\S 6)$, we see

$$
h^{1}\left(Z_{\Gamma, X}, \mathcal{O}_{Z_{\Gamma, X}}\right)=h^{2}\left(X_{\Gamma}, \mathcal{O}_{X_{\Gamma}}\left(-Z_{\Gamma, X}\right)\right)=h^{1}\left(X_{\Gamma}, \mathcal{O}_{X_{\Gamma}}\left(-(a+2) F_{\Gamma}\right)\right) .
$$

Then the ideal sequence of $(a+2)$ general members of $\left|F_{\Gamma}\right|$

$$
0 \longrightarrow \mathcal{O}_{X_{\Gamma}}\left(-(a+2) F_{\Gamma}\right) \longrightarrow \mathcal{O}_{X_{\Gamma}} \longrightarrow \mathcal{O}_{(a+2) F_{\Gamma}} \longrightarrow 0
$$

yields $h^{0}\left(\mathcal{O}_{(a+2) F_{\Gamma}}\right)-1 \leqslant 1$, hence $a \leqslant 0$.

As $E_{\Gamma} \cdot \xi_{a-b}=a=0$, the image $Z_{X}$ of $Z_{\Gamma, X}$ is still contractible. We can even explicitly give the supporting divisor: denote the image of $F_{\Gamma}$ in $X$ by $F$. They are Cartier, as $\Gamma \subset X_{\text {reg. The }}$ supporting divisor is $H=Z_{X}+F \in \operatorname{Pic}(X)$, which is big and nef. Indeed, $\sigma^{*} H=Z_{\Gamma, X}+F_{\Gamma}+E_{\Gamma}$. As $Z_{\Gamma, X}+F_{\Gamma}=\varphi^{*}\left(\xi_{1}+\mathfrak{f}\right)$ is nef, and $\sigma^{*} H$ restricted to $E_{\Gamma}$ is trivial, $H$ is nef. A direct computation shows $H^{3}=1$. By the base point free theorem, $|k H|$ is free for $k \gg 0$, defining a birational contraction $\phi: X \longrightarrow V$, contracting $Z_{X}$ to a curve. The base locus $\Gamma \subset Z_{X}$ is contracted to a point, the general fiber of the elliptic pencil on $Z_{X}$ is a section. The variety $V$ is again a Gorenstein Fano threefold with canonical singularities and $K_{X}=\phi^{*} K_{V}+Z_{X}$. From $\phi^{*} K_{V}=K_{X}-Z_{X}=-2 H$ we conclude that $-K_{V}$ is divisible by 2 in $\operatorname{Pic}(V)$. From $H^{0}(X, k H)=1+\frac{k}{6}\left(8+3 k+k^{2}\right)$ we see that $V$ is a sextic in $\mathbb{P}\left(1^{3}, 2,3\right)$.

Proposition 8.2. If $W \simeq \mathbb{P}_{1} \times \mathbb{P}_{1}$, then $X \simeq \mathbb{P}_{1} \times S_{1}$, where $S_{1}$ denotes a normal del Pezzo surface of degree 1 (and $a=b=0, m=4$ ).

Proof. In this case, $Z_{\Gamma, X}$ is the pullback of one ruling of $W=\mathbb{P}_{1} \times \mathbb{P}_{1}$. The general fiber of $Z_{\Gamma, X}$ is a smooth elliptic curve, and $Z_{\Gamma, X}$ meets the singular locus of $X_{\Gamma}$ at most in points. Going from $X_{\Gamma}$ to $Y_{\Gamma}$, we see $a \leqslant 0$. As $E_{\Gamma} \simeq W$, we have $a=b$, and $X$ Fano implies that $a=b=0$. As $\varphi$ followed by the natural projection $W \rightarrow \mathbb{P}_{1}$ contracts all the fibers of $\sigma: X_{\Gamma} \rightarrow X$ to points, we obtain an induced map $X \longrightarrow \mathbb{P}_{1}$ with general fiber $F=\sigma\left(F_{\Gamma}\right)$ and section $\Gamma$, where $F$ is a normal del Pezzo surface of degree one. We have $-K_{X_{\Gamma}}=Z_{\Gamma, X}+2 F_{\Gamma}$. As above, $-K_{X}=Z_{X}+2 F$, and we see that $Z_{X}$ is nef, so $\left|k Z_{X}\right|$ is free for $k \gg 0$. The map defined by $\left|k Z_{X}\right|$ is a $\mathbb{P}_{1}$-bundle with section $F$ and fiber $\Gamma$. As in [Isk80] we conclude that $X \simeq F \times \mathbb{P}_{1}$ is a product.

\section{ACKNOWLEDGEMENTS}

The authors gratefully acknowledge support by the Schwerpunkt program Globale Methoden in der komplexen Geometrie of the Deutsche Forschungsgemeinschaft. They also want to thank the referee for many valuable remarks and comments.

\section{REFERENCES}

Ben85 X. Benveniste, Sur le cone des 1-cycles effectifs en dimension 3, Math. Ann. 272 (1985), 257-265.

Ber07 E. Bertini, Introduzione alla geometria proiettiva degli iperspazi (Enrico Spoerri, Pisa, 1907).

Che99 I. A. Chel'tsov, Three-dimensional Fano varieties of integer index, Math. Notes 66 (1999), 360-365.

Cut88 S. Cutkosky, Elementary contractions of Gorenstein threefolds, Math. Ann. 280 (1988), 521-525.

delP85 P. del Pezzo, Sulle superficie di ordine $n$ immerse nello spazio di $n+1$ dimensioni, Rend. R. Acc. delle Scienze Fisiche e Mat. di Napoli 24 (1885), 212-216. 


\section{Gorenstein FAno threefolds With BASE POINTS}

DPS93 J.-P. Demailly, T. Peternell and M. Schneider, Kähler manifolds with numerically effective Ricci class, Compositio Math. 89 (1993), 217-240.

IT01 S. Ishii and M. Tomari, Hypersurface non-rational singularities which look canonical from their Newton boundaries, Math. Z. 237 (2001), 125-147.

Isk78 V. A. Iskovskikh, Fano 3-folds I, II, Math. USSR Izv. 11 (1977), 485-527; 12 (1978), 469-506.

Isk80 V. A. Iskovskikh, Anticanonical models of three-dimensional algebraic varieties, J. Soviet Math. 13 (1980), 745-814.

IP99 V. A. Iskovskikh and Yu. G. Prokhorov, Algebraic geometry V: Fano varieties (Springer, Berlin, 1999).

Kaw88 Y. Kawamata, Crepant blowing-up of 3-dimensional canonical singularities and its application to degenerations of surfaces, Ann. of Math. (2) 127 (1988), 93-163.

KM98 J. Kollár and S. Mori, Birational geometry of algebraic varieties (Cambridge University Press, Cambridge, 1998).

Mel99 M. Mella, Existence of good divisors on Mukai varieties, J. Algebraic Geom. 8 (1999), 197-206.

Mor82 S. Mori, Threefolds whose canonical bundles are not numerically effective, Ann. of Math. (2) 116 (1982), 133-176.

Mor88 S. Mori, Lectures on 3-dimensional algebraic varieties, University of Utah, Fall 1988.

Rei79 M. Reid, Canonical 3-folds, in Algebraic geometry, Angers, 1979, ed. A. Beauville (Sijthoff \& Noordhoff, Alphen aan den Rijn, 1980), 273-310.

Rei83 M. Reid, Projective morphisms according to Kawamata, unpublished manuscript (1983).

Sai74 B. Saint-Donat, Projective models of K3 surfaces, Amer. J. Math. 96 (1974), 602-639.

Shi89 K.-H. Shin, 3-dimensional Fano varieties with canonical singularities, Tokyo J. Math. 12 (1989), 375-385.

Sho80 V. V. Shokurov, Smoothness of a general anticanonical divisor on a Fano variety, Math. USSR Izv. 14 (1980), 395-405.

Priska Jahnke priska.jahnke@uni-bayreuth.de

Mathematisches Institut, Universität Bayreuth, D-95440 Bayreuth, Germany

Ivo Radloff ivo.radloff@uni-bayreuth.de

Mathematisches Institut, Universität Bayreuth, D-95440 Bayreuth, Germany 